\title{
Experimental and Theory Study of the Lossless Storage Law for Marine LNG Storage Tank Based on Modified Three-District Model
}

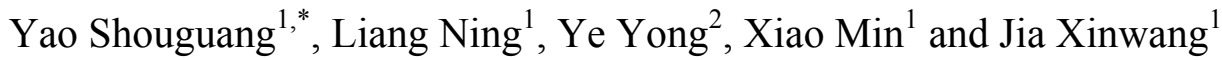 \\ ${ }^{I}$ School of Energy and Power Engineering, Jiangsu University of Science and Technology, Zhenjiang, 2120031, P.R. \\ China; ${ }^{2}$ CSSC Shenghui Equipment CO, LTD. Zhangjiagang 215632, P.R. China
}

\begin{abstract}
In this paper a Modified Three-District Model was introduced to study the marine medium-sized horizontal LNG storage tank. The horizontal cryogenic tank evaporation rate and the lossless storage time with the liquid nitrogen were experimented. The maximum error of the pressure change and the lossless storage time obtained by the modified Three-District Model and by the experiment was observed to be only about $4.7 \%$. So the applicability and reliability of the modified model for marine medium-sized horizontal LNG storage tank have been verified. Furthermore, based on the marine horizontal LNG storage tank with an effective volume of $250 \mathrm{~m}^{3}$ in a dual fuel-filled barge, the forecast analysis on the pressure change under different conditions of fullness rate, environmental temperature and heat leakage has also been discussed.
\end{abstract}

Keywords: Calculation model, Cryogenic tank, Experiment research, Heat leakage, Pressure change, The lossless storage.

\section{INTRODUCTION}

In view of the importance of lossless storage in cryogenic liquid storage and transportation process, since the 1980s, a variety of models have been put forward to simulate the thermal process in the container, and many calculation programs have been done to predict the performance of cryogenic vessels [1-4]. But now, most researches have been focussing on the small and medium-sized vertical cryogenic storage tank with an initial fullness of not more than $90 \%$. Under the condition of the initial rate of $95 \%$, medium-sized marine horizontal cryogenic storage tank differs from the vertical storage tanks in the existence of an unstable region near the bottom of the tank in the horizontal cryogenic tank. Besides, under certain conditions, the boundary layer will be separated from the concave wall. In addition, the dynamic changes of the heat leakage in the vapor space are not considered in the traditional thermodynamic model, resulting in large deviations in the later boost [5]. On the basis of that, a modified Three-District Model storage calculation model has been introduced in this paper. The applicability and reliability of the established model were validated by experiments on marine medium-sized horizontal cryogenic storage tanks. While the laws of the lossless storage on LNG cryogenic storage tank in the effective volume of $250 \mathrm{~m}^{3}$ were also studied by the established model.

\section{RESEARCH OBJECT}

The structure of the studied horizontal cryogenic tanks is made up of cryogenic insulation bivalve, which are supported by the saddle bearing. Geometric volume is $263.2 \mathrm{~m}^{3}$,

*Address correspondence to this author at the School of Energy and Power Engineering, Jiangsu University of Science and Technology, Zhenjiang, 2120031, P.R. China, CSSC Shenghui Equipment CO, LTD. Zhangjiagang 215632,P.R. China; E-mail: zjyaosg@126.com the effective volume is $250 \mathrm{~m}^{3}$, and the value of inner pot surface area is $299.9 \mathrm{~m}^{2}$, and the insulation is in the form of vacuum powder. The basic structure of the tank is shown in Fig. (1).

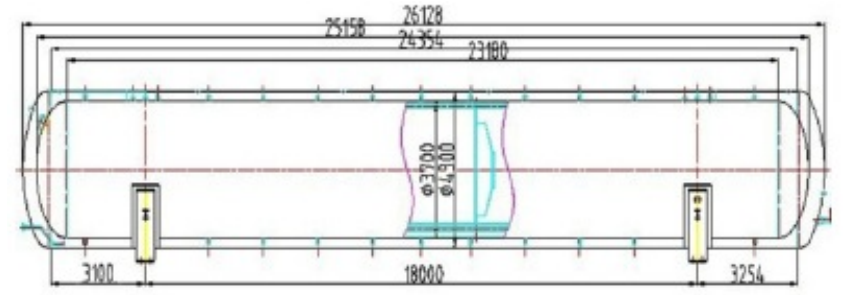

Fig. (1). The basic structure of the tank.

\section{MODIFIED THREE-REGION MODEL}

Heat flow processes in the horizontal cryogenic storage tank is shown in Fig. (2). The outside heat mainly enters into the tank from the contact points on the tank wall surrounded by low temperature liquid. The liquid near the thin layer rises along the tank wall after being heated. The liquid forms a boundary layer alongside the wall with its upward movement. At the same time the heat which is constantly leaking is absorbed into the tank from the outside environment. And when the layer develops up to the intersect interface of the liquid and vapor, the liquid then flows to the central area. After which, the cryogenic liquid starts to converge. Similarly, the cryogenic liquid at the bottom will also move upward, and merges with the liquid mentioned above, forming a circulation process within the storage tank. Similar to the liquid space, the natural convections of vapors on the wall and liquid-vapor interface also exist in the vapor space.

The main assumptions for building the lossless storage model in cryogenic tank are expressed as follows $[5,6]$ : 


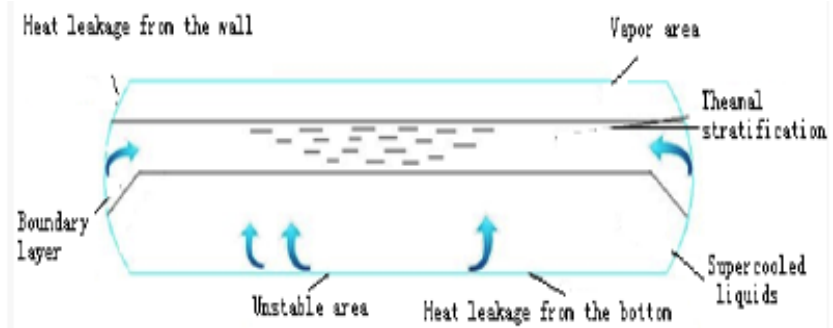

Fig. (2). Heat flow processes in the horizontal cryogenic storage tank.

(1) On the basis of three-domain model and boundary layer theory, the fluids in cryogenic tank are divided into four parts: the vapor phase space, the thermal stratification region, the main body area, the boundary layer.

(2) The total heat leakage per unit time for the cryogenic tank is assumed to have a constant value. The uneven heat leakage due to the tank structure is ignored, and the heat flux densities are assumed to be the same in all parts of the tank. So the heat leakage of the vapor phase space and liquid phase space can be calculated by the theory of vapor phase space.

(3) The nucleate boiling cannot be formed due to the low heat flux density of the heat leakage. The flow state of tank fluids and boundary layer are turbulent flow.

(4) There is a vapor-liquid interface between the vapor phase space and thermal stratification region. That the temperature is the saturation temperature under the corresponding pressure, and no volume and quality exist on the vaporliquid interface, are also assumed.

(5) The temperature in the vapor phase space, the thermal stratification region and the main body area are all uniform. Vapor phase space is filled with the overheating vapor. Besides, the main body area is occupied by the super-cooled liquid. Furthermore the temperature in thermal stratification region is higher than the main body area.

(6) The fluid boundary layer is supposed to be stable, and the quality of inflow through the boundary layer per unit time equals to the outflow; meanwhile the portion flow fluids was heated by the absorbed heat.

(7) The absorbed heat is calculated by the demarcation point between start point and off point. The absorbed heat from fluid boundary is used for evaporation of cryogenic liquid and heating of thermal stratification region, while the residual heat is used for heating up the main body.

The liquid nitrogen in the horizontal tank is selected as the research object. Q is used to express the total heat leakage unit time. The process of heat and mass transfer in four regions of cryogenic liquid can be shown as follows:

(1) The total energy conservation equation:

$$
Q=q_{l} \cdot F_{w l}+q_{g} \cdot F_{w g}
$$

Where $q_{l}, q_{g}$ represent the heat leakage of the liquid phase space and vapor phase space in per unit area and per unit time in the storage tank, respectively, W/m2; $F_{w l}$ and
$F_{w g}$ represent the contact area of the internal face with liquid phase space and the vapor phase space, $\mathrm{m}^{2}$.

Relationship of the heat leakage per unit area and per unit time between the liquid phase space and vapor phase space is decided by the heat leakage theory of the vapor phase space [7]:

(2) Energy conservation equation in boundary layer

$$
m_{t d l} \cdot h_{t i n}-m_{t d l} \cdot h_{t o u t}+F_{w t d l} \cdot q_{l}=0
$$

Where, $m_{t d l}$ stands for the fluid quality flowing into or out of the boundary layer per unit time; $h_{\text {tin }}$ is the fluid enthalpy flowing into the fluid boundary layer, $J / \mathrm{kg} ; h_{\text {tout }}$ is the fluid enthalpy flowing out the fluid boundary layer, $J / \mathrm{kg} ; F_{\text {wtdl }}$ is the contact area between the fluid boundary layer and internal face, $m^{2}$, whose value is associated with the starting point and off point of the fluid boundary. If the boundary layer does not exist, $F_{w t d l}$ is equal to zero.

(3) Mass conservation equation of the main body area

$$
\frac{\partial}{\partial \tau}\left(\rho_{t m b} V_{t m b}\right)=m_{t d l}
$$

Where, $\rho_{t m b}$ is the density of main fluids in the storage tank, $\mathrm{kg}_{3} / \mathrm{m}^{3} ; V_{t m b}$ is the volume of main fluids in the storage tank, $m^{3}$.

(4) Energy conservation equation of the main body area

$$
\frac{\partial}{\partial \tau}\left(\rho_{t m b} V_{t m b} h_{t i n}\right)=F_{w t m b} q_{l}
$$

Where $F_{w t m b}$ is the direct contact area between the internal face of the storage tank and the main fluid. The formula is $F_{w t m b}=F_{w l}-F_{w t d l}, m^{2}$.

(5) Liquid evaporation on vapor-liquid interface is:

$$
G_{f}=G_{f 1}+G_{f 2}
$$

Where, $G f_{1}$ is the natural evaporation rate on the vaporliquid interface by the high temperature liquid in the boundary layer. $G f_{2}$ means the evaporation liquid rate which is caused by the vapor-liquid two-phase heat exchange on vapor-liquid interface. Specific formula processing (see Ref [8]).

(6) Mass conservation of the thermal stratification region is

$$
\frac{\partial}{\partial \tau}\left(\rho_{t s} V_{t s}\right)=m_{t d l}-G_{f}
$$

Where, $\rho_{t s}$ is the density of thermal stratification region, $\mathrm{kg} / \mathrm{m}^{3} ; V_{t s}$ is the volume of thermal stratification region, $\mathrm{m}^{3}$.

(7) Energy conservation of the thermal stratification region is

$$
\frac{\partial}{\partial \tau}\left(\rho_{t s} V_{t s} h_{t s}\right)=\left(m_{t d l}-G_{f}\right) \cdot h_{s}
$$

Where, $h_{t s}$ stands for the enthalpy of thermal stratification region, $\mathrm{J} / \mathrm{kg}$.

(8) Mass conservation equation of the vapor phase space is:

$$
\frac{\partial}{\partial \tau}\left(\rho_{g} V_{g}\right)=G_{f}
$$


Where, $\rho_{g}$ is the density of vapor in vapor phase space, $\mathrm{kg} / \mathrm{m}^{3} ; V_{g}$ is the volume of vapor phase space, $\mathrm{m}^{3}$.

(9) Energy conservation equation of the vapor in vapor phase space

$$
\begin{aligned}
& \frac{\partial}{\partial \tau}\left(\rho_{g} V_{g} h_{g}\right)= \\
& F_{w g} \cdot q_{g}-Q_{f}+G_{f 1} h_{s}^{\prime \prime}+G_{f 2} \cdot h_{g}
\end{aligned}
$$

Where, $h_{s}$ s represents the enthalpy of saturated vapor under the saturation temperature at the vapor-liquid interface, $\mathrm{J} / \mathrm{kg}$.

Other equations include the total volume conservation equation, working medium state equation, and the tank geometry parameter equation. The set the temperature of the liquid fluid boundary layer is higher than the saturation temperature, $0.1 \mathrm{~K}$, which forms a complete analysis and calculation model.

\section{EXPRIMENTAL STUDIES}

According to the national standard GB/T18443.5, the steam flow rate method was selected to measure the flow rate of evaporation vapor, temperature and pressure at equilibrium in this experiment, and the evaporation rate of cryogenic vessel was calculated under the condition of standard [9].

Experimental measurement system is shown in Fig. (3). Firstly, the fullness rate was taken as $95 \%$. Experimental data was recorded every hour, and continuously for 48 hours after the system became stable. Then the storage was filled with liquid nitrogen with a fullness rate of $95 \%$ again after completing the above experiment. All valves of storage tank were closed and the vapor vaporizer, thermometer, mass flow meter were cut off. After this, the lossless storage experiment was started. During the experiment the data acquisition instrument was used to record the value of the changing pressure and temperature. The lossless storage experiment was ended when the value of pressure in the storage tank reached 1.2 $\mathrm{MPa}$.

For the experiments on the static evaporation rates and the lossless storage on the same horizontal liquid nitrogen storage tank with the volume of $250 \mathrm{~m}^{3}$, the data of

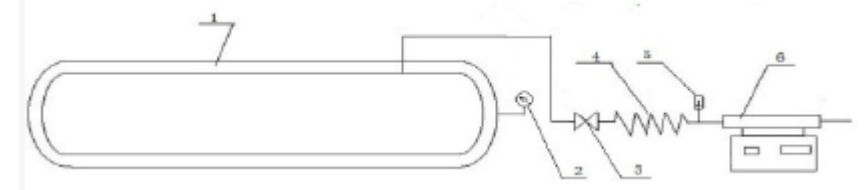

1-Checked storage tank; 2-Differential pressure level meter; 3-Vent valve; 4-Carburetor; 5-Thermometer; 6-Vapor mass flow meter

Fig. (3). Measuring device schematic diagram.

The daily average evaporation rate and storage tank evaporation rate through the experiment are shown in Table 1 and Table 2, and in the lossless storage experiment, the changes of pressure in storage tank with time was shown in Fig. (4).
Table 1. Daily average evaporation rate in the process of experiment.

\begin{tabular}{|c|c|c|c|c|}
\hline Time & $\begin{array}{c}\text { Meter } \\
\text { Reading } \\
(\mathbf{L} / \mathbf{m})\end{array}$ & $\begin{array}{c}\text { Atmospheric } \\
\text { Pressure } \\
(\mathbf{M P a})\end{array}$ & $\begin{array}{c}\text { Meter Inlet } \\
\text { Temperature } \\
\left.\mathbf{(}{ }^{\mathbf{}} \mathbf{C}\right)\end{array}$ & $\begin{array}{c}\text { Meter Inlet } \\
\text { Pressure } \\
\mathbf{( k p a )}\end{array}$ \\
\hline \hline $\begin{array}{c}24 \text { hours } \\
\text { before }\end{array}$ & 191.4 & 0.1005 & 31.525 & 100.77 \\
\hline $\begin{array}{c}24 \text { hours } \\
\text { later }\end{array}$ & 189 & 0.1006 & 32.296 & 100.79 \\
\hline
\end{tabular}

Table 2. Experimental results of storage tank evaporation

\begin{tabular}{|c|c|c|c|}
\hline & $\begin{array}{c}\text { Test of Evapora- } \\
\text { tion Rate }\end{array}$ & $\begin{array}{c}\text { Static Evapora- } \\
\text { tion Rate }\end{array}$ & $\begin{array}{c}\text { Difference Value of } \\
\text { Static Evaporation rate }\end{array}$ \\
\hline $\begin{array}{c}24 \text { hours } \\
\text { before }\end{array}$ & $0.171 \%$ & $0.16 \%$ & \multirow{2}{*}{$\begin{array}{c}(0.1614-0.1588) / \\
0.1614 \times 100 \%=1.6 \% \\
<5 \%\end{array}$} \\
\hline $\begin{array}{c}24 \text { hours } \\
\text { later }\end{array}$ & $0.169 \%$ & $0.16 \%$ & \\
\hline
\end{tabular}
rate.

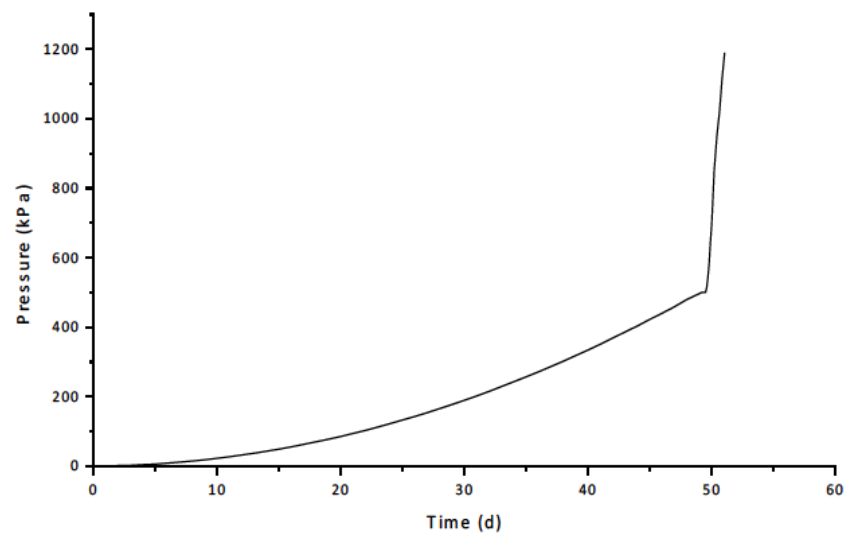

Fig. (4). Pressure changes with time during the storage in the fullness rate of $95 \%$.

Under the conditions of the experimental environment parameters, the experiment indicated that the safe storage time for liquid nitrogen in the storage tank with the effective volume of $250 \mathrm{~m}^{3}$ was 51.042 days.

According to the static evaporation rate $(0.1588 \%)$ obtained by the experiment on the low temperature liquid nitrogen storage tank, the total heat leakage per unit time for the fullness rate of $95 \%$ was $739.297 \mathrm{~W}$.

\section{COMPARISON AND ANALYSIS WITH MODIFIED MODEL}

The initial physic parameters were selected as follows: the initial ambient temperature was $305 \mathrm{~K}$, the initial ambient pressure was $101325 \mathrm{~Pa}$ and the initial fullness rate was 0.95 . The laws of lossless storage can be obtained by the calculation program mentioned above, and the comparison of the experiment and the simulation are shown in Fig. (5). 


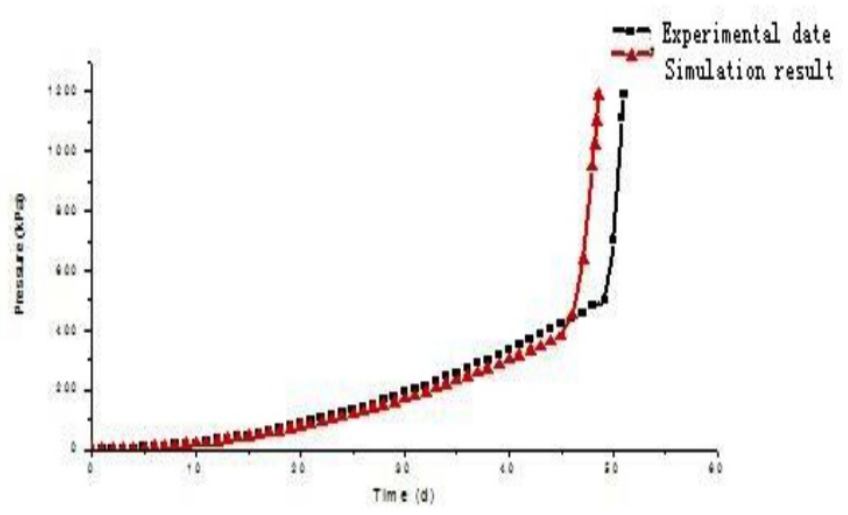

Fig. (5). Comparisons of experiment and the simulation.

From Fig. (5), it can be clearly seen that the pressure obtained by the simulation agreed well with experimental data at the beginning, which indicates that the assumption of the modified model was suitable for the actual state in the initial stage. However, with the passage of time, the simulation results and experimental data had a certain bias that gradually increased. The maximum deviation was $4.7 \%$, while the maximum deviation reached $9 \%$ by the original ThreeDistrict Model. Thus, the predication generated by the modified model is more consistent with the experimental results. In short, the modified calculation model can very well reflect the real situation of marine medium tank.

\section{Results under different initial fullness rates}

For effective volume of $250 \mathrm{~m}^{3}$ marine horizontal LNG storage tanks, the heat leakage and temperature were chosen to be $740 \mathrm{~W}$ and $305 \mathrm{~K}$ respectively. Besides, the initial rates were taken to be $95 \%, 80 \%, 70 \%$ and $60 \%$. The results calculated by the modified model are shown in Fig. (6).

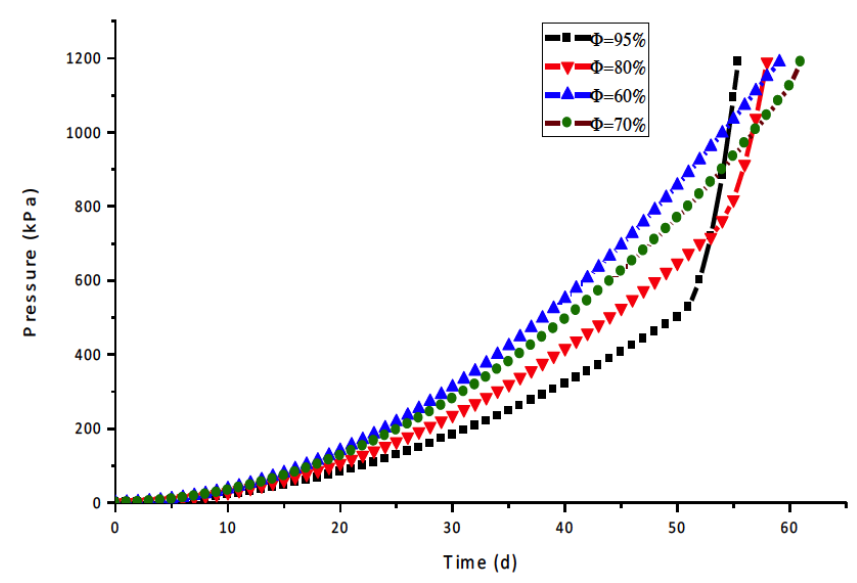

Fig. (6). Laws of lossless storage under different fullness rates.

Fig. (6) shows that for the cryogenic LNG horizontal tank, with the increase of the initial fullness rate, the lossless storage time is reduced after the first increase. In the initial fullness rate of near $70 \%$, the lossless storage time can be the longest, so the best initial fullness rate should be between $70 \%$ and $80 \%$. When the initial fullness rate is less than $70 \%$, the liquid in the tank is relatively small, so under the same heat leakage, the temperature will rise faster. However if the fullness rate is larger than $80 \%$, even in the early stage, the pressure rises slowly, but with the temperature of the liquid in the tank increasing, the density will decrease, which in turn will drastically reduce the volume occupied by the vapors, and the pressure will suddenly increase. Meanwhile it can also be seen that at the initial stage the curve of the low fullness rate was at the top, which means that the pressure increases faster than that of the large fullness rate. This is because the obtained internal energy of the liquid and cryogenic vapor is mainly from the heat leakage. Besides the vapor, the internal energy in per unit volume is far less than that of the liquid due to its small density. Therefore the pressure will rise more slowly in the same heat leakage, because there is more liquid than the less one.

\section{Results under different heat leakages}

Initial environmental pressure was taken as $101325 \mathrm{~Pa}$, and the initial temperature was $305 \mathrm{k}$, meanwhile the heat leakages were selected as $740 \mathrm{~W}, 640 \mathrm{~W}, 540 \mathrm{~W}, 400 \mathrm{~W}$, the results were calculated by the modified model and are shown in Fig. (7).

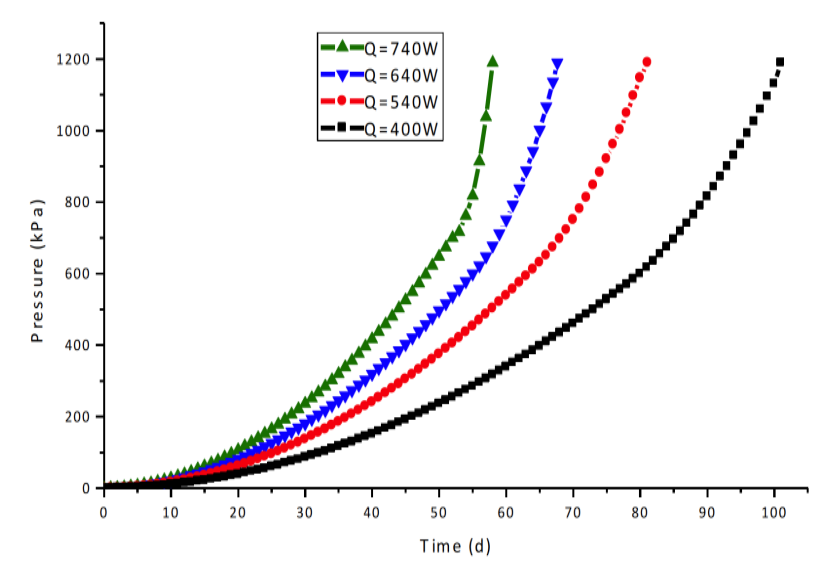

Fig. (7). Laws of lossless storage under different heat leakages.

As can be seen from Fig. (7), when the tank structure and the initial fullness rate remain constant, the lossless storage showed a similar rule under different heat leakages. The larger heat leakage only accelerated the rate of the rising pressure, but the law of pressure remains unchanged.

\section{Calculation results under different ambient tempera- tures}

The values of initial environmental pressure and heat leakages were $101325 \mathrm{~Pa}$ and $740 \mathrm{w}$ respectively. The initial temperatures were selected as $320 \mathrm{~K}, 305 \mathrm{~K}$, and $290 \mathrm{~K}$. The modified non-destructive storage calculation model was used to get the results, as shown in Fig. (8).

As can be seen from the Fig. (8), the environment temperature also has a certain influence on the length of the lossless storage time. In general, the lower the temperature, the longer the storage time, on the contrary the higher the temperature, the shorter the storage time. So for safety reasons, it is suggested that in the actual production, the destructive experiments of cryogenic storage tanks should be done in the summer ambient temperatures as much as possible. 


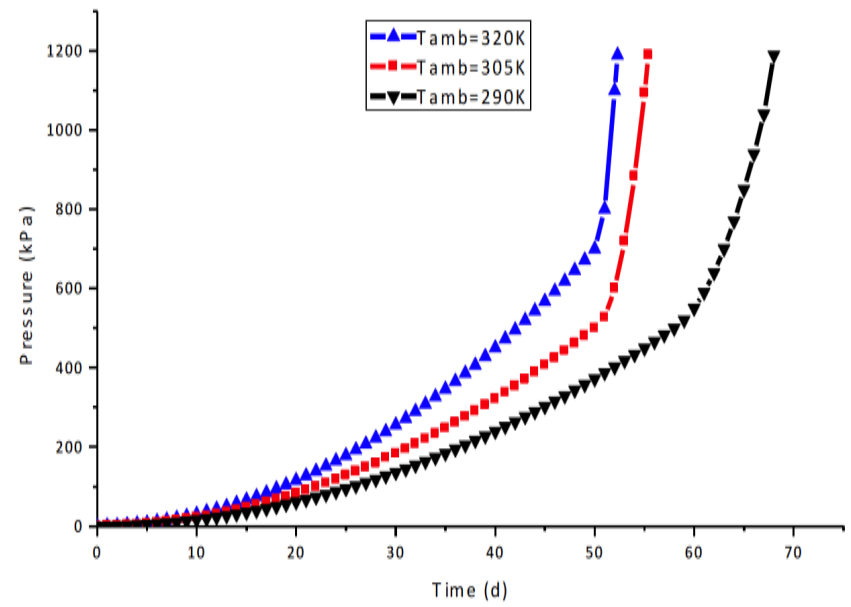

Fig. (8). Laws of lossless storage under different ambient temperatures.

\section{CONCLUSION}

For the marine medium-sized horizontal LNG storage tank, the study of the rate of static evaporation and the rule of lossless storage can draw the following two conclusions:

1. By the experimental verification, modified three-region calculation model introduced in this paper, was applied to the marine medium-sized horizontal LNG storage tank and was found to be appropriate. The forecast analysis of the rate of evaporation and the rule of lossless storage has high accuracy.

2. In view of the effective volume of $250 \mathrm{~m}^{3}$ Marine horizontal LNG storage tank, the results of the forecast analysis of lossless storage rule provided a good foundation data for the enterprise's product development and design of security storage and transportation scheme.

\section{CONFLICT OF INTEREST}

The authors confirm that this article content has no conflict of interest.

\section{ACKNOWLEDGEMENTS}

Declared none.

\section{REFERENCES}

[1] Q.S. Chen, J. Wegrzyn, and V. Prasad, "Analysis of temperature and pressure changes in liquefied natural gas(LNG) cryogenic tank", Cryogenics, vol. 44, no. 10, pp. 701-709, 2004.

[2] J. Si, "Performances of non-loss storage for horizontal multi-layer insulation vessel", Cryogenics, vol. 4, pp. 39-41, 2006.

[3] B. Song, Y. Shi, and R. Wang, "Progress on foreign studies of the ventless storage of cryogenic liquid", Cryogenics and Superconductivity, vol. 35, no. 6, pp. 469-473, 2007.

[4] X. Chen, and C. Su "Saturation phase thermodynamics model research of no-loss storage and rising tank", Cryogenics and Superconductivity, vol. 39, no. 7, pp. 13-20, 2011.

[5] C.M. Yu, N.U. Aydemir, and J.E.S. Venart, "Transient convection and thermal stratification in uniformly-heated partially-filled horizontal cylindrical and spherical vessels", Journal of Thermal Science, vol. 1, no. 2, pp. 115-122, 1992.

[6] B. Song, "Study on the Rule of Ventless Storage in Cryogenic Tank", China: Shanghai Jiao Tong University, 2008.

[7] B. Song, Y Shi, and R. Wang, "The analysis of the heat leakage rate in gas space in a cryogenic tank", Cryogenics and Superconductivity, vol. 36, no. 4, pp. 18-22, 2008.

[8] N. Liang, The Theory and Experimental Research of Lossless Storage Rule for Marine Horizontal LNG Cryogenic Tank, China: Zhenjiang Jiangsu University of Science and Technology, 2014.

[9] GB/T18443.5-2001 Testing method of cryo-insulation pressure vessels: Available from: http://www.chinesestandard.net/PDFEnglish-Translation/GBT18443.5-2001.html

(c) Shouguang et al.; Licensee Bentham Open.

This is an open access article licensed under the terms of the Creative Commons Attribution Non-Commercial License (http://creativecommons.org/licenses/by-nc/4.0/) which permits unrestricted, non-commercial use, distribution and reproduction in any medium, provided the work is properly cited. 